PROCEEDINGS OF THE

AMERICAN MATHEMATICAL SOCIETY

Volume 131, Number 8, Pages 2505-2514

S 0002-9939(02)06891-0

Article electronically published on November 27, 2002

\title{
ON A PROPOSED CHARACTERIZATION OF SCHATTEN-CLASS COMPOSITION OPERATORS
}

\author{
JINGBO XIA
}

(Communicated by Joseph A. Ball)

\begin{abstract}
For an analytic function $\varphi$ which maps the open unit disc $D$ to itself, let $C_{\varphi}$ be the operator of composition with $\varphi$ on the Bergman space $L_{a}^{2}(D, d A)$. It has been a longstanding problem to determine whether or not the membership of $C_{\varphi}$ in the Schatten class $\mathcal{C}_{p}, 1<p<\infty$, is equivalent to the condition that the function $z \mapsto\left\{\left(1-|z|^{2}\right) /\left(1-|\varphi(z)|^{2}\right)\right\}^{p}$ has a finite integral with respect to the Möbius-invariant measure $d \lambda(z)=\left(1-|z|^{2}\right)^{-2} d A(z)$ on $D$. We show that the answer is negative when $2<p<\infty$.
\end{abstract}

\section{INTRODUCTION}

Let $D$ be the open unit disc $\{z \in \mathbf{C}:|z|<1\}$ in the complex plane and let $d A$ be the area measure on $D$ normalized in such a way that $A(D)=1$. Throughout the paper, $d \lambda$ denotes the Möbius-invariant measure on $D$, i.e.,

$$
d \lambda(z)=\left(1-|z|^{2}\right)^{-2} d A(z) .
$$

Recall that the Bergman space $L_{a}^{2}(D, d A)$ is defined to be $\left\{f \in L^{2}(D, d A): f\right.$ is analytic on $D\}$. For an analytic function $\varphi: D \rightarrow D$, let $C_{\varphi}$ denote the operator of composition with $\varphi$ on $L_{a}^{2}(D, d A)$. That is,

$$
\left(C_{\varphi} f\right)(z)=f(\varphi(z)), \quad f \in L_{a}^{2}(D, d A) .
$$

It is a consequence of Littlewood's subordination theorem that the operator $C_{\varphi}$ is bounded on $L_{a}^{2}(D, d A)$ for any analytic function $\varphi: D \rightarrow D$ [7, Theorem 10.3.2].

Recall that, for any $1 \leq p<\infty$, the Schatten $p$-class $\mathcal{C}_{p}$ consists of operators $T$ satisfying the condition $\|T\|_{p}<\infty$, where the $p$-norm is defined by the formula

$$
\|T\|_{p}=\left\{\operatorname{tr}\left(|T|^{p}\right)\right\}^{1 / p}=\left\{\operatorname{tr}\left(\left(T^{*} T\right)^{p / 2}\right)\right\}^{1 / p} .
$$

The main interest of this paper concerns a proposed characterization of the membership of $C_{\varphi}$ in $\mathcal{C}_{p}$. Let us briefly review the relevant background for the benefit of the reader, even though the problem itself is well known. In [5], D. Luecking and K. Zhu proved that $C_{\varphi} \in \mathcal{C}_{p}$ if and only if the function $z \mapsto\{\log (1 /|z|)\}^{-2} N_{\varphi, 2}(z)$ belongs to $L^{p / 2}(D, d \lambda)$, where $d \lambda$ is given by (1.1) and $N_{\varphi, 2}(z)=\sum_{w \in \varphi^{-1}\{z\}}\{\log (1 /|w|)\}^{2}$, which is a generalized Nevanlinna counting function for $\varphi$. But such a characterization of the membership $C_{\varphi} \in \mathcal{C}_{p}$ was not considered to be completely satisfactory

Received by the editors March 22, 2002.

2000 Mathematics Subject Classification. Primary 47B10, 47B33, 47B38.

This work was supported in part by National Science Foundation grant DMS-0100249. 
(see [7, page 226]) because $\varphi$ enters in an indirect way, namely through the counting function $N_{\varphi, 2}$. An alternate criterion for $C_{\varphi} \in \mathcal{C}_{p}$, one which not only involves $\varphi$ directly but also appears to be aesthetically more pleasing, was proposed and debated in [4, 5, 7]. More precisely, the following problem arose (in chronological order) on page 363 of [4], on pages 226-228 of [7], and in Section 7 of [5].

Problem 1. Let $1<p<\infty$ and let $\varphi: D \rightarrow D$ be an analytic function. Is it true that the composition operator $C_{\varphi}: L_{a}^{2}(D, d A) \rightarrow L_{a}^{2}(D, d A)$ belongs to the Schatten class $\mathcal{C}_{p}$ if and only if

$$
\int_{D}\left(\frac{1-|z|^{2}}{1-|\varphi(z)|^{2}}\right)^{p} d \lambda(z)<\infty ?
$$

There are several reasons for proposing this problem. First of all,

$$
\left\{\left(1-|z|^{2}\right) /\left(1-|\varphi(z)|^{2}\right)\right\}^{p}=\left\{\left\langle C_{\varphi} C_{\varphi}^{*} k_{z}, k_{z}\right\rangle\right\}^{p / 2},
$$

where $k_{z}(w)=\left(1-|z|^{2}\right) /(1-\bar{z} w)^{2}$ (see [7, Proposition 10.3.4]) and the function $z \mapsto\left\langle C_{\varphi} C_{\varphi}^{*} k_{z}, k_{z}\right\rangle=\left\|C_{\varphi}^{*} k_{z}\right\|^{2}$ is the Berezin symbol of $C_{\varphi} C_{\varphi}^{*}$. Secondly, it is known that $C_{\varphi}$ is compact if and only if

$$
\lim _{|z| \uparrow 1} \frac{1-|z|^{2}}{1-|\varphi(z)|^{2}}=0
$$

(see [6] or [1, Theorem 3.22]). Thirdly, (1.2) is sufficient for $C_{\varphi} \in \mathcal{C}_{p}$ when $1<p \leq 2$ and necessary for $C_{\varphi} \in \mathcal{C}_{p}$ when $2 \leq p<\infty$ (see [5, Section 7]). In particular, $C_{\varphi} \in \mathcal{C}_{2}$ if and only if (1.2) holds with $p=2$. Thus, when $2<p<\infty$, (1.2) appears to be the natural "interpolation" between the compactness criterion and the Hilbert-Schmidt-class criterion for $C_{\varphi}$. Furthermore, Zhu recently proved the following:

Theorem 2 ([8 Theorem 1.1]). Let $2 \leq p<\infty$. Let $\varphi: D \rightarrow D$ be an analytic function which has bounded valence, i.e., there is a positive integer $N$ such that $\operatorname{card}\{w \in D: \varphi(w)=z\} \leq N$ for every $z \in D$. Then $C_{\varphi} \in \mathcal{C}_{p}$ if and only if (1.2) holds.

The purpose of this paper is to report that, however pleasing or natural (1.2) may appear, this condition in fact is not sufficient for the membership $C_{\varphi} \in \mathcal{C}_{p}$ when $2<p<\infty$. Thus Luecking's comment on page 363 of [4 turns out to be prophetic after all. The following is our main result:

Theorem 3. For any $2<p<\infty$, there exists an analytic function $\varphi: D \rightarrow D$ such that

$$
\int_{D}\left(\frac{1-|z|^{2}}{1-|\varphi(z)|^{2}}\right)^{p} d \lambda(z)<\infty
$$

and such that the composition operator $C_{\varphi}: L_{a}^{2}(D, d A) \rightarrow L_{a}^{2}(D, d A)$ does not belong to the Schatten class $\mathcal{C}_{p}$.

This result leads to the following contrast between the Berezin symbol of $C_{\varphi}^{*} C_{\varphi}$ and the Berezin symbol of $C_{\varphi} C_{\varphi}^{*}$ which seems to be interesting. Consider the conditions
(a) $\int_{D}\left\|C_{\varphi} k_{z}\right\|^{p} d \lambda(z)<\infty$
(b) $\int_{D}\left\|C_{\varphi}^{*} k_{z}\right\|^{p} d \lambda(z)<\infty$. 
Because $\left\|C_{\varphi} k_{z}\right\|^{2}$ is the Berezin symbol of $C_{\varphi}^{*} C_{\varphi}$ and because $C_{\varphi}^{*} C_{\varphi}$ is a Toeplitz operator as defined on page 106 of [7], condition (a) implies $C_{\varphi} \in \mathcal{C}_{p}$ [8] Lemmas 2.1 and 2.2]. On the other hand, as we have already mentioned, the Berezin symbol of $C_{\varphi} C_{\varphi}^{*}$ equals $\left\|C_{\varphi}^{*} k_{z}\right\|^{2}=\left(1-|z|^{2}\right)^{2} /\left(1-|\varphi(z)|^{2}\right)^{2}$. Theorem 3 tells us that condition (b) does not imply $C_{\varphi} \in \mathcal{C}_{p}$ when $2<p<\infty$. Thus conditions (a) and (b) are not equivalent.

The proof of Theorem 3 is technical but self-contained. In fact, the only thing the reader needs to known about $L_{a}^{2}(D, d A)$ is that $\left\{(\ell+1)^{1 / 2} z^{\ell}: \ell=0,1,2, \ldots\right\}$ is an orthonormal basis. To ensure that (1.3) holds, we only need to control the modulus $|\varphi|$ of $\varphi$. Thanks to a new lower bound for $\operatorname{tr}\left(\left(C_{\varphi}^{*} C_{\varphi}\right)^{p / 2}\right)$ (see (3.4) below), our proof that $C_{\varphi} \notin \mathcal{C}_{p}$ also involves the modulus $|\varphi|$ only. But the main obstacle in the proof of Theorem 3 lies in the fact that it is difficult to prescribe the modulus of an analytic function. In other words, the analyticity requirement is a severe handicap for the construction of $\varphi$. Fortunately, by carefully exploiting the distribution of the Poisson kernel, we are able to find just enough control on $D$ to make such a construction possible.

The rest of the paper is organized as follows. In Section 2 we construct the required $\varphi$ and establish the estimates which are necessary for the proof of Theorem 3. The proof itself is completed in Section 3.

\section{Construction and estimates}

The construction of the desired $\varphi$ begins with the intervals

$$
T_{n}=\left(2^{-(n+1)}, 2^{-n}\right], \quad S_{n}=\left((4 / 3) 2^{-(n+1)},(5 / 3) 2^{-(n+1)}\right]
$$

in $\mathbf{R}, n=1,2, \ldots$. That is, $S_{n}$ is the middle third of $T_{n}$. Let $t_{n}=(4 / 3) 2^{-(n+1)}$, which is the left end-point of $S_{n}, n \in \mathbf{N}$.

Let $2<p<\infty$ be given. We choose a rational number $\epsilon$ such that

$$
0<\epsilon<p^{-1} \text {. }
$$

The requirement $\epsilon<p^{-1}$ ensures $\lim _{k \rightarrow \infty} 2^{-\left(p^{-1}-\epsilon\right) k}=0$. This allows us to choose a strictly increasing sequence $k(1)<\ldots<k(n)<\ldots$ of positive integers such that

$$
2^{-\left(p^{-1}+\epsilon\right) k(n)} \cdot 2 \cdot 2^{2 \epsilon k(n)} \leq(1 / 3) 2^{-(n+1)}
$$

for every $n \in \mathbf{N}$ and such that every $\epsilon k(n)$ is an integer. While the requirement that both inequalities in (2.1) be strict is indispensable to the proof of Theorem 3 , the stipulation that $\epsilon$ be rational, which makes it possible for $\epsilon k(n)$ to be an integer, is not. But the fact that $\epsilon k(n)$ is chosen to be an integer does allow us to avoid certain unnecessary trivialities.

Next we subdivide $S_{n}$. For integers $n \geq 1$ and $1 \leq j \leq 2^{2 \epsilon k(n)}$, define the intervals

$$
\begin{aligned}
& J_{n, j}=\left(t_{n}+2^{-\left(p^{-1}+\epsilon\right) k(n)} \cdot 2 \cdot(j-1), t_{n}+2^{-\left(p^{-1}+\epsilon\right) k(n)} \cdot 2 \cdot j\right), \\
& I_{n, j}=\left(t_{n}+2^{-\left(p^{-1}+\epsilon\right) k(n)} \cdot 2 \cdot(j-1), t_{n}+2^{-\left(p^{-1}+\epsilon\right) k(n)} \cdot(2 j-1)\right),
\end{aligned}
$$

where, as we recall, $t_{n}$ is the left end-point of $S_{n}$. Thus, $I_{n, j}$ is the left half of $J_{n, j}$ and the $J_{n, j}$ 's are pairwise disjoint. (2.2) ensures that $\bigcup_{j=1}^{2^{2 \epsilon k(n)}} J_{n, j} \subset S_{n}$. Keep in mind that the length of $I_{n, j}$ equals $2^{-\left(p^{-1}+\epsilon\right) k(n)}$. We now define a measurable 
function $u$ on the unit circle $T=\{\tau \in \mathbf{C}:|\tau|=1\}$ as follows:

$$
\begin{aligned}
& u\left(e^{i t}\right)=2^{-k(n)} \quad \text { if } t \in \bigcup_{j=1}^{2^{2 \epsilon k(n)}} I_{n, j}, n \geq 1, \\
& u\left(e^{i t}\right)=1 \quad \text { if } t \in(-\pi, \pi] \backslash\left\{\bigcup_{n=1}^{\infty} \bigcup_{j=1}^{2^{2 \epsilon k(n)}} I_{n, j}\right\} .
\end{aligned}
$$

The harmonic extension of $u$ to $D$ will be denoted by the same symbol. Finally, define

$$
\begin{aligned}
& h(z)=\frac{1}{2 \pi} \int_{-\pi}^{\pi} \frac{e^{i t}+z}{e^{i t}-z} u\left(e^{i t}\right) d t, \\
& \varphi(z)=\exp (-h(z)), \quad z \in D
\end{aligned}
$$

Obviously, $\operatorname{Re}\{h(z)\}=u(z)>0$ for every $z \in D$. Therefore $\varphi$ maps $D$ to itself. Our remaining task is to verify that $\varphi$ satisfies (1.3) and has the property $C_{\varphi} \notin \mathcal{C}_{p}$. This verification is based on a number of estimates of the modulus $|\varphi(z)|$, which will take up the rest of the section.

We begin with the Poisson kernel $P(z ; \tau)=\left(1-|z|^{2}\right) /|1-z \bar{\tau}|^{2}, \tau \in T$ and $z \in D$. There exist constants $0<\alpha<\beta<\infty$ such that if $1 / 2 \leq r<1$ and $|\theta-t| \leq 5$, then

$$
\frac{\alpha(1-r)}{(1-r)^{2}+(\theta-t)^{2}} \leq \frac{1}{2 \pi} P\left(r e^{i \theta} ; e^{i t}\right) \leq \frac{\beta(1-r)}{(1-r)^{2}+(\theta-t)^{2}} .
$$

This follows from the identity $\left|1-r e^{i(\theta-t)}\right|^{2}=(1-r)^{2}+2 r(1-\cos (\theta-t))$ and the fact that there are $0<a<b<\infty$ such that $a \leq x^{-2}(1-\cos x) \leq b$ when $0<x \leq 5$.

Lemma 4. For any $n \in \mathbf{N}$ and $1 \leq j \leq 2^{2 \epsilon k(n)}$, define

$$
G_{n, j}=\left\{r e^{i \theta}: \theta \in I_{n, j}, 0<1-r \leq 2^{-\left(p^{-1}+\epsilon\right) k(n)}\right\} .
$$

Then there is a constant $C_{4}$ which is independent of $n, j$ such that

$$
\int_{G_{n, j}} \frac{(1-|z|)^{p-2}}{(1-|\varphi(z)|)^{p}} d A(z) \leq C_{4} 2^{-\epsilon p k(n)}
$$

Proof. Given such a pair of $n, j$, we have $G_{n, j}=\bigcup_{\nu=0}^{k(n)} G_{n, j}^{\nu}$, where

$$
\begin{aligned}
& G_{n, j}^{0}=\left\{r e^{i \theta}: \theta \in I_{n, j}, 0<1-r \leq 2^{-\left(1+p^{-1}+\epsilon\right) k(n)}\right\}, \\
& G_{n, j}^{\nu}=\left\{r e^{i \theta}: \theta \in I_{n, j}, 2^{-\left(1+p^{-1}+\epsilon\right) k(n)} \cdot 2^{(\nu-1)}<1-r \leq 2^{-\left(1+p^{-1}+\epsilon\right) k(n)} \cdot 2^{\nu}\right\}
\end{aligned}
$$

for $1 \leq \nu \leq k(n)$. Since $|\varphi(z)|=e^{-\operatorname{Re}\{h(z)\}}=e^{-u(z)}$, the proof hinges on an estimate of $u(z)$ on each $G_{n, j}^{\nu}$. For this purpose let us write $I_{n, j}=\left(a_{n, j}, b_{n, j}\right)$ and $J_{n, j}=\left(a_{n, j}, c_{n, j}\right)$. Then $c_{n, j}-b_{n, j}=b_{n, j}-a_{n, j}=2^{-\left(p^{-1}+\epsilon\right) k(n)}$, which will be denoted by $\rho_{n}$. Let us first consider the case $z \in G_{n, j}^{\nu}$ where $1 \leq \nu \leq k(n)$. For such a $z$, write $z=r e^{i \theta}$ with $\theta \in I_{n, j}$. Since $u\left(e^{i t}\right)=1$ for $t \in J_{n, j} \backslash I_{n, j}=\left[b_{n, j}, c_{n, j}\right)$, 
recalling (2.4), we have

$$
\begin{aligned}
u\left(r e^{i \theta}\right) & \geq \frac{1}{2 \pi} \int_{J_{n, j} \backslash I_{n, j}} P\left(r e^{i \theta} ; e^{i t}\right) d t \geq \alpha \int_{b_{n, j}}^{c_{n, j}} \frac{1-r}{(1-r)^{2}+(t-\theta)^{2}} d t \\
& \geq \alpha \int_{b_{n, j}}^{c_{n, j}} \frac{1-r}{(1-r)^{2}+\left(t-a_{n, j}\right)^{2}} d t \\
& =\alpha \int_{\rho_{n}}^{2 \rho_{n}} \frac{1-r}{(1-r)^{2}+s^{2}} d s=\alpha \int_{\rho_{n} /(1-r)}^{2 \rho_{n} /(1-r)} \frac{d x}{1+x^{2}} .
\end{aligned}
$$

The condition $r e^{i \theta} \in G_{n, j}^{\nu}$ implies $1-r \leq 2^{-\left(1+p^{-1}+\epsilon\right) k(n)} \cdot 2^{\nu}=\rho_{n} \cdot 2^{-k(n)+\nu}$. In particular, $\rho_{n} /(1-r) \geq 1$. Hence $\left(1+x^{2}\right)^{-1} \geq\left(2 x^{2}\right)^{-1}$ if $x \geq \rho_{n} /(1-r)$. The condition $r e^{i \theta} \in G_{n, j}^{\nu}$ also requires $2^{-\left(1+p^{-1}+\epsilon\right) k(n)} \cdot 2^{(\nu-1)}<1-r$, i.e., $2^{-k(n)+\nu-1}<$ $(1-r) / \rho_{n}$. Thus

$$
u\left(r e^{i \theta}\right) \geq \frac{\alpha}{2} \int_{\rho_{n} /(1-r)}^{2 \rho_{n} /(1-r)} \frac{1}{x^{2}} d x=\frac{\alpha}{4} \cdot \frac{1-r}{\rho_{n}} \geq \frac{\alpha}{4} 2^{-k(n)+\nu-1}=\frac{\alpha}{8} 2^{-k(n)+\nu}
$$

if $r e^{i \theta} \in G_{n, j}^{\nu}$ and $1 \leq \nu \leq k(n)$.

Now suppose that $r e^{i \theta} \in G_{n, j}^{0}$, where $\theta \in I_{n, j}$. In this case we use the fact that $u\left(e^{i t}\right) \geq 2^{-k(n)}$ for $t \in T_{n}$. Obviously, $\left[\theta-\rho_{n}, \theta+\rho_{n}\right] \subset T_{n}$. Therefore

$$
\begin{aligned}
u\left(r e^{i \theta}\right) & \geq 2^{-k(n)} \frac{1}{2 \pi} \int_{\theta-\rho_{n}}^{\theta+\rho_{n}} P\left(r e^{i \theta} ; e^{i t}\right) d t \geq 2^{-k(n)} \alpha \int_{\theta-\rho_{n}}^{\theta+\rho_{n}} \frac{1-r}{(1-r)^{2}+(t-\theta)^{2}} d t \\
& =2^{-k(n)} \alpha \int_{-\rho_{n}}^{\rho_{n}} \frac{1-r}{(1-r)^{2}+s^{2}} d s \geq 2^{-k(n)} \alpha \int_{-1}^{1} \frac{d x}{1+x^{2}},
\end{aligned}
$$

where the last $\geq$ is due to the fact that $1-r \leq 2^{-\left(1+p^{-1}+\epsilon\right) k(n)}=2^{-k(n)} \rho_{n}$.

Combining (2.7) with (2.6), we see that there is a constant $0<c<1$ which is independent of $n, j$ such that $u(z) \geq c 2^{-k(n)+\nu}$ if $z \in G_{n, j}^{\nu}$ and $0 \leq \nu \leq k(n)$. This implies that $1-|\varphi(z)|=1-e^{-u(z)} \geq 1-\exp \left(-c 2^{-k(n)+\nu}\right)$ if $z \in G_{n, j}^{\nu}$ and $0 \leq \nu \leq k(n)$. Thus, if we let $\delta=\inf _{0<x \leq 1} x^{-1}\left(1-e^{-x}\right)$, then

$$
\inf _{z \in G_{n, j}^{\nu}}(1-|\varphi(z)|)^{p} \geq(\delta c)^{p} \cdot 2^{-p k(n)} \cdot 2^{p \nu}, \quad 0 \leq \nu \leq k(n) .
$$

Because $p-2>0$, it follows from the definition of $G_{n, j}^{\nu}$ that

$$
\sup _{z \in G_{n, j}^{\nu}}(1-|z|)^{p-2} \leq 2^{-(p-2)\left(1+p^{-1}+\epsilon\right) k(n)} \cdot 2^{(p-2) \nu}, \quad 0 \leq \nu \leq k(n) .
$$

From the definitions of $G_{n, j}^{\nu}$ and $I_{n, j}$ it obviously follows that

$$
A\left(G_{n, j}^{\nu}\right) \leq 2^{-\left(p^{-1}+\epsilon\right) k(n)} \cdot 2^{-\left(1+p^{-1}+\epsilon\right) k(n)} \cdot 2^{\nu}, \quad 0 \leq \nu \leq k(n) .
$$

Combining (2.8)-(2.10) and simplifying the exponents involved, we find that

$$
\int_{G_{n, j}^{\nu}} \frac{(1-|z|)^{p-2}}{(1-|\varphi(z)|)^{p}} d A(z) \leq \frac{\sup _{z \in G_{n, j}^{\nu}}(1-|z|)^{p-2}}{\inf _{z \in G_{n, j}^{\nu}}(1-|\varphi(z)|)^{p}} A\left(G_{n, j}^{\nu}\right) \leq(c \delta)^{-p} \cdot 2^{-\nu} \cdot 2^{-\epsilon p k(n)}
$$

for all $0 \leq \nu \leq k(n)$. Recall that $G_{n, j}=\bigcup_{\nu=0}^{k(n)} G_{n, j}^{\nu}$. Because of the factor $2^{-\nu}$ that appears on the right-hand side of (2.11), if we sum both sides of this inequality as $\nu$ ranges from 0 to $k(n)$, we see that the conclusion of the lemma holds for $C_{4}=2(c \delta)^{-p}$. 
Lemma 5. For any $n \geq 1$ and $1 \leq j \leq 2^{2 \epsilon k(n)}$, let $B_{n, j}$ be the middle third of $I_{n, j}$. That is, $B_{n, j}=\left(3^{-1}\left(b_{n, j}+2 a_{n, j}\right), 3^{-1}\left(2 b_{n, j}+a_{n, j}\right)\right)$, where $a_{n, j}<b_{n, j}$ are the end-points of $I_{n, j}$. Furthermore, for such $n$ and $j$, define

$$
E_{n, j}=\left\{r e^{i t}: t \in B_{n, j}, 0<1-r \leq 2^{-\left(1+p^{-1}+\epsilon\right) k(n)}\right\} .
$$

Then $\sup _{z \in E_{n, j}} u(z) \leq(1+6 \beta) 2^{-k(n)}$, where $\beta$ is the constant that appears in (2.4).

Proof. Let $n \geq 1$ and $1 \leq j \leq 2^{2 \epsilon k(n)}$ be given. Consider an arbitrary $z=r e^{i \theta} \in$ $E_{n, j}$, where $\theta \in B_{n, j}$. Since $B_{n, j}$ is the middle third of $I_{n, j}$, we have $|\theta-t| \geq \rho_{n} / 3$ if $t \in \mathbf{R} \backslash I_{n, j}$, where $\rho_{n}=2^{-\left(p^{-1}+\epsilon\right) k(n)}$ is the length of $I_{n, j}$. The condition $\theta \in B_{n, j}$ also implies $0<\theta<1 / 2$. Therefore $(-\pi, \pi] \backslash I_{n, j} \subset\left\{t \in \mathbf{R}: \rho_{n} / 3 \leq|\theta-t| \leq \pi+\right.$ $(1 / 2)\}$. Now, because $u\left(e^{i t}\right)=2^{-k(n)}$ for $t \in I_{n, j}$ and $u\left(e^{i t}\right) \leq 1$ for $t \in(-\pi, \pi] \backslash I_{n, j}$, we have

$$
\begin{aligned}
u\left(r e^{i \theta}\right) & =\frac{1}{2 \pi} \int_{-\pi}^{\pi} P\left(r e^{i \theta} ; e^{i t}\right) u\left(e^{i t}\right) d t \leq 2^{-k(n)}+\frac{1}{2 \pi} \int_{(-\pi, \pi] \backslash I_{n, j}} P\left(r e^{i \theta} ; e^{i t}\right) d t \\
& \leq 2^{-k(n)}+\beta \int_{\rho_{n} / 3 \leq|\theta-t| \leq \pi+(1 / 2)} \frac{1-r}{(1-r)^{2}+(\theta-t)^{2}} d t,
\end{aligned}
$$

where $\beta$ is the constant that appears in (2.4). But

$$
\begin{aligned}
\int_{|\theta-t| \geq \rho_{n} / 3} \frac{1-r}{(1-r)^{2}+(\theta-t)^{2}} d t & =2 \int_{\rho_{n} / 3}^{\infty} \frac{1-r}{(1-r)^{2}+s^{2}} d s \\
& =2 \int_{\rho_{n} / 3(1-r)}^{\infty} \frac{1}{1+x^{2}} d x \\
& \leq 6(1-r) / \rho_{n} .
\end{aligned}
$$

The condition $r e^{i \theta} \in E_{n, j}$ requires $1-r \leq 2^{-\left(1+p^{-1}+\epsilon\right) k(n)}=2^{-k(n)} \rho_{n}$. Thus it follows from (2.13) and (2.14) that $u(z) \leq(1+6 \beta) 2^{-k(n)}$ for every $z \in E_{n, j}$.

Lemma 6. Let $U=\bigcup_{n=1}^{\infty} \bigcup_{j=1}^{2^{2 \epsilon k(n)}} I_{n, j}$. Then for any $x \in \mathbf{R} \backslash U$ and $0<a<\infty$, we have

$$
m((x-a, x+a) \backslash U) \geq(2 / 3) a,
$$

where $m$ is the standard Lebesgue measure on $\mathbf{R}$.

Proof. The case where either $(x-a, x) \cap U=\emptyset$ or $(x, x+a) \cap U=\emptyset$ is trivial. Thus let us assume that $x \in \mathbf{R} \backslash U$ and $a>0$ are such that $(x-a, x) \cap U \neq \emptyset$ and $(x, x+a) \cap U \neq \emptyset$. Because $x \notin U$, this means that there are at least two intervals in the family

$$
\mathcal{F}=\left\{I_{n, j}:(x-a, x+a) \cap I_{n, j} \neq \emptyset, n \geq 1,1 \leq j \leq 2^{2 \epsilon k(n)}\right\} .
$$

Let $Y$ be the smallest open interval such that

$$
Y \cap U=(x-a, x+a) \cap U .
$$

Since $(x-a, x+a) \backslash U=\{Y \backslash U\} \cup\{(x-a, x+a) \backslash Y\}$, (2.15) will follow once we show that $m(Y \backslash U) \geq(1 / 3) m(Y)$.

From the arrangement $I_{n, j} \subset S_{n} \subset T_{n}=\left(2^{-(n+1)}, 2^{-n}\right]$ it is easy to see that we can re-enumerate the family $\mathcal{F}$ as $\left\{\left(a_{\nu}, b_{\nu}\right): 1 \leq \nu<N\right\}$, where $3 \leq N \leq \infty$, such that $b_{\nu+1}<a_{\nu}$ for all $1 \leq \nu<N-1$. Thus $Y \cap U=\bigcup_{1 \leq \nu<N} I_{\nu}$, where $I_{1}=\left(a_{1}, b_{1}^{\prime}\right)$ with $a_{1}<b_{1}^{\prime} \leq b_{1}, I_{\nu}=\left(a_{\nu}, b_{\nu}\right)$ if $2 \leq \nu<N-1$, and, in the 
event $N<\infty, I_{N-1}=\left(a_{N-1}^{\prime}, b_{N-1}\right)$ with $a_{N-1} \leq a_{N-1}^{\prime}<b_{N-1}$. The definition of the $I_{n, j}$ 's ensures that the length of the gap between $\left(a_{\nu+1}, b_{\nu+1}\right)$ and $\left(a_{\nu}, b_{\nu}\right)$ is greater than or equal to the length of $\left(a_{\nu}, b_{\nu}\right)$. That is, $a_{\nu}-b_{\nu+1} \geq b_{\nu}-a_{\nu}$, $1 \leq \nu<N-1$. Also, $b_{\nu}-a_{\nu} \geq b_{\nu+1}-a_{\nu+1}$ by the definition of the $I_{n, j}$ 's. Since $Y \backslash U=\bigcup_{1 \leq \nu<N-1}\left[b_{\nu+1}, a_{\nu}\right]$, we have

$$
\begin{aligned}
m(Y \backslash U) & =\sum_{1 \leq \nu<N-1}\left(a_{\nu}-b_{\nu+1}\right) \geq \sum_{1 \leq \nu<N-1}\left(b_{\nu}-a_{\nu}\right) \\
& \geq \frac{1}{2} \sum_{1 \leq \nu<N}\left(b_{\nu}-a_{\nu}\right) \geq \frac{1}{2} m(Y \cap U) .
\end{aligned}
$$

Adding $(1 / 2) m(Y \backslash U)$ to both sides, we find that $(3 / 2) m(Y \backslash U) \geq(1 / 2) m(Y)$. Thus $m(Y \backslash U) \geq(1 / 3) m(Y)$ as promised.

Lemma 7. There is a $c_{7}>0$ such that

$$
u(z) \geq c_{7} \quad \text { for every } z \in D \backslash\left\{\bigcup_{n=1}^{\infty} \bigcup_{j=1}^{2 \epsilon k(n)} G_{n, j}\right\},
$$

where $G_{n, j}$ is defined by (2.5).

Proof. Let $W=\left\{r e^{i t}: 3 / 4<r<1, t \in(-1 / 4,3 / 4)\right\}$. Since $u\left(e^{i t}\right)=1$ when $t \in$ $(-\pi, \pi] \backslash(0,1 / 2]$, we have $\lim _{r \uparrow 1} u\left(r e^{i t}\right)=1$ uniformly for $t \in(-\pi, \pi] \backslash(-1 / 8,5 / 8)$. Hence it suffices to find a $c_{7}>0$ such that

$$
u(z) \geq c_{7} \quad \text { for all } z \in W \backslash\left\{\bigcup_{n=1}^{\infty} \bigcup_{j=1}^{2^{2 \epsilon k(n)}} G_{n, j}\right\} .
$$

For any $0 \leq r<1$ and $\theta \in \mathbf{R}$, define $I(\theta, r)=(\theta-3(1-r), \theta+3(1-r))$. Then

$$
\frac{1-r}{(1-r)^{2}+(\theta-t)^{2}}=\frac{1}{1-r} \cdot\left\{1+\left(\frac{\theta-t}{1-r}\right)^{2}\right\}^{-1} \geq \frac{10^{-1}}{1-r} \chi_{I(\theta, r)}(t) .
$$

Let $\theta \in(-1 / 4,3 / 4)$ and $3 / 4<r<1$. Then $I(\theta, r) \subset(-\pi, \pi]$ and $u\left(e^{i t}\right)=1$ for $t \in I(\theta, r) \backslash U$, where $U=\bigcup_{n=1}^{\infty} \bigcup_{j=1}^{2 \epsilon k(n)} I_{n, j}$ as in Lemma 6. By (2.4) and (2.16),

$$
\begin{aligned}
u\left(r e^{i \theta}\right) & =\frac{1}{2 \pi} \int_{-\pi}^{\pi} P\left(r e^{i \theta} ; e^{i t}\right) u\left(e^{i t}\right) d t \geq \frac{\alpha}{10} \cdot \frac{1}{1-r} \int_{I(\theta, r)} u\left(e^{i t}\right) d t \\
& \geq \frac{\alpha}{10} \cdot \frac{m(I(\theta, r) \backslash U)}{1-r} .
\end{aligned}
$$

Let us further assume $r e^{i \theta} \in W \backslash\left\{\bigcup_{n=1}^{\infty} \bigcup_{j=1}^{2^{2 \epsilon k(n)}} G_{n, j}\right\}$. We consider the following two cases:

(i) If $\theta \in(-1 / 4,3 / 4) \backslash U$, then we apply Lemma 6 to the case where $x=\theta$ and $a=3(1-r)$ to obtain

$$
\begin{aligned}
m(I(\theta, r) \backslash U) & =m((\theta-3(1-r), \theta+3(1-r)) \backslash U) \\
& \geq(2 / 3) \cdot 3(1-r)=2(1-r) .
\end{aligned}
$$

By (2.17), we have $u\left(r e^{i \theta}\right) \geq \alpha / 5$ in this case.

(ii) If $\theta \in U$, then there exist an $n \geq 1$ and a $1 \leq j \leq k(n)$ such that $\theta \in I_{n, j}$. Now, because $r e^{i \theta} \notin G_{n, j}$, (2.5) tells us $1-r>2^{-\left(p^{-1}+\epsilon\right) k(n)}=\rho_{n}$, 
the length of $I_{n, j}$. Since the distance between $\theta$ and $J_{n, j} \backslash I_{n, j}$ is less than $\rho_{n}$, we can pick a $\theta^{\prime} \in J_{n, j} \backslash I_{n, j}$ such that $\left|\theta-\theta^{\prime}\right|<\rho_{n}<1-r$. Thus $I(\theta, r)=$ $(\theta-3(1-r), \theta+3(1-r)) \supset\left(\theta^{\prime}-2(1-r), \theta^{\prime}+2(1-r)\right)$. Since $J_{n, j} \backslash I_{n, j} \subset \mathbf{R} \backslash U$, we now apply Lemma 6 to the case where $x=\theta^{\prime}$ and $a=2(1-r)$ to obtain $m(I(\theta, r) \backslash U) \geq m\left(\left(\theta^{\prime}-2(1-r), \theta^{\prime}+2(1-r)\right) \backslash U\right) \geq(2 / 3) \cdot 2(1-r)$. By $(2.17)$, we have $u\left(r e^{i \theta}\right) \geq 2 \alpha / 15$ in this case. This completes the proof.

\section{Proof of Theorem 3}

We must show that the analytic function $\varphi: D \rightarrow D$ defined by (2.3) satisfies (1.3) and has the property that $C_{\varphi} \notin \mathcal{C}_{p}$. Let us first verify (1.3).

Let $G=\bigcup_{n=1}^{\infty} \bigcup_{j=1}^{2 \epsilon k(n)} G_{n, j}$, where $G_{n, j}$ is given by (2.5). By Lemma $7,|\varphi(z)|=$ $e^{-\operatorname{Re}\{h(z)\}}=e^{-u(z)} \leq e^{-c_{7}}$ when $z \in D \backslash G$. Therefore

$$
\int_{D \backslash G}\left(\frac{1-|z|^{2}}{1-|\varphi(z)|^{2}}\right)^{p} d \lambda(z)=\int_{D \backslash G} \frac{\left(1-|z|^{2}\right)^{p-2}}{\left(1-|\varphi(z)|^{2}\right)^{p}} d A(z)<\infty .
$$

Invoking Lemma 4, we have

$$
\begin{aligned}
& \int_{G}\left(\frac{1-|z|^{2}}{1-|\varphi(z)|^{2}}\right)^{p} d \lambda(z) \leq \sum_{n=1}^{\infty} \sum_{j=1}^{2^{2 \epsilon k(n)}} \int_{G_{n, j}} \frac{2^{p-2}(1-|z|)^{p-2}}{(1-|\varphi(z)|)^{p}} d A(z) \\
& \leq 2^{p-2} C_{4} \sum_{n=1}^{\infty} 2^{-\epsilon p k(n)} \cdot 2^{2 \epsilon k(n)}=2^{p-2} C_{4} \sum_{n=1}^{\infty} 2^{-\epsilon(p-2) k(n)}
\end{aligned}
$$

Because $p>2$ and $\epsilon>0$, the above is finite and (1.3) follows from (3.1) and (3.2).

To show that $C_{\varphi} \notin \mathcal{C}_{p}$ or, what amounts to the same, $\operatorname{tr}\left(\left(C_{\varphi}^{*} C_{\varphi}\right)^{p / 2}\right)=\infty$, we need the following inequality: For any $1<\rho<\infty$ and $0<x<1$, we have

$$
\sum_{\ell=0}^{\infty}(\ell+1)^{\rho} x^{\ell} \geq \frac{1}{(1-x)^{\rho+1}} .
$$

The proof of (3.3) is elementary. We begin with the identity

$$
(1-x)^{-2}=\sum_{\ell=0}^{\infty}(\ell+1) x^{\ell}=\sum_{\ell=0}^{\infty}\left\{(\ell+1)^{\rho} x^{\ell}\right\}^{1 / \rho} \cdot\left\{x^{\ell}\right\}^{(\rho-1) / \rho}
$$

and apply Hölder's inequality with conjugate exponents $\rho$ and $\rho /(\rho-1)$. This gives us $(1-x)^{-2} \leq\left(\sum_{\ell=0}^{\infty}(\ell+1)^{\rho} x^{\ell}\right)^{1 / \rho}(1-x)^{-(\rho-1) / \rho}$. Multiplying both sides by $(1-x)^{(\rho-1) / \rho}$, we find that $(1-x)^{-(\rho+1) / \rho} \leq\left(\sum_{\ell=0}^{\infty}(\ell+1)^{\rho} x^{\ell}\right)^{1 / \rho}$. Clearly, (3.3) follows from this.

Let $e_{\ell}(z)=(\ell+1)^{1 / 2} z^{\ell}, \ell=0,1,2, \ldots$. . Recall that $\left\{e_{\ell}: \ell \geq 0\right\}$ is the standard orthonormal basis for the Bergman space $L_{a}^{2}(D, d A)$. Because $p / 2>1$ and $\left\|e_{\ell}\right\|=1$, it follows from the spectral decomposition of $C_{\varphi}^{*} C_{\varphi}$ and Hölder's inequality that $\left\langle\left(C_{\varphi}^{*} C_{\varphi}\right)^{p / 2} e_{\ell}, e_{\ell}\right\rangle \geq\left\{\left\langle C_{\varphi}^{*} C_{\varphi} e_{\ell}, e_{\ell}\right\rangle\right\}^{p / 2}=\left\|C_{\varphi} e_{\ell}\right\|^{p}$ [7, Proposition 6.3.3]. But $C_{\varphi} e_{\ell}=(\ell+1)^{1 / 2} \varphi^{\ell}$. Therefore

$$
\left\langle\left(C_{\varphi}^{*} C_{\varphi}\right)^{p / 2} e_{\ell}, e_{\ell}\right\rangle \geq(\ell+1)^{p / 2}\left\|\varphi^{\ell}\right\|^{p} \geq(\ell+1)^{p / 2}\left\{\sum_{n=1}^{\infty} \int_{E_{n}}|\varphi|^{2 \ell} d A\right\}^{p / 2}
$$


where $E_{n}=\bigcup_{j=1}^{2^{2 \epsilon k(n)}} E_{n, j}, n \in \mathbf{N}$, and $E_{n, j}$ is defined by (2.12). Define

$$
f_{n}=\inf _{z \in E_{n}}|\varphi(z)|, \quad n \in \mathbf{N}
$$

It is elementary that $\left\{\sum_{n} a_{n}\right\}^{\rho} \geq \sum_{n} a_{n}^{\rho}$ if $\rho>1$ and $a_{n} \geq 0$. Hence

$$
\begin{aligned}
\left\langle\left(C_{\varphi}^{*} C_{\varphi}\right)^{p / 2} e_{\ell}, e_{\ell}\right\rangle & \geq(\ell+1)^{p / 2}\left\{\sum_{n=1}^{\infty} f_{n}^{2 \ell} A\left(E_{n}\right)\right\}^{p / 2} \\
& \geq(\ell+1)^{p / 2} \sum_{n=1}^{\infty}\left(f_{n}^{2 \ell} A\left(E_{n}\right)\right)^{p / 2} .
\end{aligned}
$$

Therefore

$$
\begin{aligned}
\operatorname{tr}\left(\left(C_{\varphi}^{*} C_{\varphi}\right)^{p / 2}\right) & =\sum_{\ell=0}^{\infty}\left\langle\left(C_{\varphi}^{*} C_{\varphi}\right)^{p / 2} e_{\ell}, e_{\ell}\right\rangle \geq \sum_{\ell=0}^{\infty}(\ell+1)^{p / 2} \sum_{n=1}^{\infty}\left(f_{n}^{2 \ell} A\left(E_{n}\right)\right)^{p / 2} \\
& =\sum_{n=1}^{\infty}\left(A\left(E_{n}\right)\right)^{p / 2} \sum_{\ell=0}^{\infty}(\ell+1)^{p / 2}\left(f_{n}^{p}\right)^{\ell} \geq \sum_{n=1}^{\infty} \frac{\left(A\left(E_{n}\right)\right)^{p / 2}}{\left(1-f_{n}^{p}\right)^{1+(p / 2)}}
\end{aligned}
$$

where the second $\geq$ follows from an application of (3.3) with $\rho=p / 2$. Thus the conclusion $C_{\varphi} \notin \mathcal{C}_{p}$ will follow once we show that there is a $d>0$ such that

$$
\frac{\left(A\left(E_{n}\right)\right)^{p / 2}}{\left(1-f_{n}^{p}\right)^{1+(p / 2)}} \geq d \quad \text { for every } n \geq 1 .
$$

To prove (3.5), we estimate $A\left(E_{n}\right)$ and $1-f_{n}^{p}$. It follows from (2.12) that $A\left(E_{n, j}\right) \geq(2 \pi)^{-1} 2^{-\left(1+p^{-1}+\epsilon\right) k(n)} m\left(B_{n, j}\right)=(6 \pi)^{-1} 2^{-(1+(2 / p)+2 \epsilon) k(n)}$. Hence

$$
A\left(E_{n}\right)=\sum_{j=1}^{2^{2 \epsilon k(n)}} A\left(E_{n, j}\right) \geq \frac{1}{6 \pi} 2^{-(1+(2 / p)+2 \epsilon) k(n)} \cdot 2^{2 \epsilon k(n)}=\frac{1}{6 \pi} 2^{-(1+(2 / p)) k(n)}
$$

Since $|\varphi(z)|=e^{-u(z)}$, it follows from Lemma 5 that $|\varphi(z)| \geq \exp \left(-M 2^{-k(n)}\right)$ for every $z \in E_{n}$, where $M=1+6 \beta$. That is, $f_{n} \geq \exp \left(-M 2^{-k(n)}\right)$. Therefore

$$
1-f_{n}^{p} \leq 1-\exp \left(-p M 2^{-k(n)}\right) \leq p M 2^{-k(n)},
$$

where the second $\leq$ follows from the inequality $x^{-1}\left(1-e^{-x}\right) \leq 1$ for $0<x<$ $\infty$, which holds because the function $x \mapsto x^{-1}\left(1-e^{-x}\right)$ is decreasing on $(0, \infty)$. Combining (3.6) and (3.7), we see that (3.5) holds for $d=(p M)^{-1-(p / 2)}(6 \pi)^{-p / 2}$. This completes the proof of Theorem 3.

\section{REFERENCES}

1. C. Cowen and B. MacCluer, Composition operators on spaces of analytic functions, CRC Press, Boca Raton, 1995. MR 97i:47056

2. J. Garnett, Bounded analytic functions, Academic Press, New York-London, 1981. MR 83g:30037

3. S. Li, Trace ideal criteria for composition operators on Bergman spaces, Amer. J. Math. 117 (1995), 1299-1323. MR 96g:47023

4. D. Luecking, Trace ideal criteria for Toeplitz operators, J. Funct. Anal. 73 (1987), 345-368. MR 88m:47046

5. D. Luecking and K. Zhu, Composition operators belonging to the Schatten ideals, Amer. J. Math. 114 (1992), 1127-1145. MR 93i:47032

6. B. MacCluer and J. Shapiro, Angular derivatives and compact composition operators on the Hardy and Bergman spaces, Canad. J. Math. 38 (1986), 878-906. MR 87h:47048 
7. K. Zhu, Operator theory in function spaces, Marcel Dekker, New York, 1990. MR 92c:47031

8. K. Zhu, Schatten class composition operators on weighted Bergman spaces of the disk, J. Operator Theory 46 (2001), 173-181. MR 2002h:47039

Department of Mathematics, State University of New York at Buffalo, Buffalo, NEW YORK 14260

E-mail address: jxia@acsu.buffalo.edu 